\title{
INTERVENÇÃO PRECOCE NA EPILEPSIA
}

EARLY INTERVENTION IN EPILEPSY

\author{
Joaquim RAMALHO \\ Célia SILVA2 \\ Cláudia CRUZ3
}

RESUM 0: a necessidade de intervir precocemente junto de crianças em situação de risco reveste-se de fulcral importância, principalmente nos seus primeiros anos de vida, em que todas as experiências a que está sujeita condicionam todo o seu desenvolvimento posterior. Desta forma, preconiza-se que quanto mais precoce for a intervenção, maiores serão os benefícios para a criança, devida à plasticidade que uma criança de tenra idade apresenta e esta deverá ser utilizada para prevenir incapacidades secundárias. $\mathrm{O}$ presente artigo procura elaborar de um programa de intervenção na epilepsia baseado em programas educativos individuais que focam o nível de realização atual da criança, assim como a avaliação e posterior intervenção assumindo a família e a escola papéis primordiais no desenvolvimento das crianças com esta perturbação, já que se constituem como agentes impulsionadores para o sucesso dos respectivos planos de intervenção.

PALAVRAS-CHAVE: epilepsia; intervenção precoce; necessidades educativas especiais.

A BSTRACT: the need for early intervention in children who are at risk or who have disabilities is of utmost importance, especially in the early years, when all experiences they go through affect their development. Therefore, we believe that the earlier intervention takes place, the better, as it is in early years that children that human learning is enhanced, which will enables the prevention of secondary handicaps and problems. This paper includes an intervention program for children with epilepsy, regarded as a latent neurological disorder. The current plan is based on individual education programs that focus on the child's present performance, as well as on assessment and intervention, looking at family and school as playing a major role in children's development, and in the success of the respective intervention plans.

KEYWORDS: Epilepsy; Early Intervention; Special Needs.

\section{INTRODUÇÃO}

A denominação epilepsia provém do grego epilambaneim, quesignifica surpresa. É considerada uma afecção crónica, sinal ou sintoma de um transtorno neurológico (ACHARYA; HATTIANGADY; SHETTY, 2008) e manifesta-se geralmente sob a forma de crises convulsivas recorrentes cujo grau de intensidade e duração podem variar.

\footnotetext{
${ }^{1}$ Faculdade de Ciências Humanas e Sociais - Universidade Fernando Pessoa - Porto - Portugal ramalho@ufp.edu.pt

${ }^{2}$ Faculdade de Ciências Humanas e Sociais - Universidade Fernando Pessoa - Porto - Portugal 14536@ufp.edu.pt

${ }^{3}$ Faculdade de Ciências Humanas e Sociais - Universidade Fernando Pessoa - Porto - Portugal 14660@ufp.edu.pt
} 
As células cerebrais trabalham em conjunto e comunicam através de sinais elétricos. Ocasional mente dá-se um curto-circuito no cérebro e, uma parte ou mesmo todas essas células descarregam-se anormalmente, daí resultando um ataque epiléptico. Os ataques dependem das funções dos neurónios onde se dá a dita descarga eléctrica anormal, bem como se a descarga se circunscreve apenas a um grupo de células ou se propaga a outros, ou inclusive, se atinge simultaneamentetodos os neurônios. As características fundamentais da epilepsia resumem-se a uma descarga súbita excessiva, rápida da substância cinzenta, com tendência à recorrência e disfunção encefálica que se repete. Refere-se, de um modo geral, a uma perturbação ao nível da acção do cérebro, mais especificamente no sistema nervoso central e na sua atividade elétrica, que irá provocar determinadas manifestações ao nível psíquico e motor $(\mathrm{CHOl} ; \mathrm{KOH}$, 2008; FERNANDES; LI, 2006; SEPÚLVEDA, 2000).

\section{Desenvolvimento}

A primeira referência à epilepsia data de A.C. e consta também dos escritos da Grécia Antiga, porém, só durante o séc. XIX é que começou a ser estudada e aprofundada em termos científicos.

Ao longo dos tempos, as sociedades têm tido diferentes perspectivas dos indivíduos com epilepsia, já que o fato de as causas não serem totalmente conhecidas originou o surgir de inúmeros mitos e crenças que se revelaram absolutamente incorretas. Em certas culturas pensava-se que estes indivíduos possuíam poderes divinos ou mesmo demoníacos, que esta doença era contagiosa e que estava na base da deficiência mental (NIELSEN, 1999).

$\mathrm{Na}$ atualidade, embora grande parte destas crenças estejam completamente erradicadas, existem ainda outras que criam um certo estigma social eque acabam por afetar não só os portadores mas também as suas famílias.

Os estudos mais recentes têm vindo a revelar que cerca de $1 \%$ da população mundial apresenta uma forma de epilepsia (ACHARYA; $\mathrm{CHOI} ; \mathrm{KOH}$, 2008; HATTIANGADY; GOLOMB et al. 2007; SHETTY, 2008). Segundo a Liga Portuguesa Contra a Epilepsia (LPCE), a prevalência de epilepsia em Portugal é de 4 a 7 casos por cada 1000 de habitantes, o que significa que cerca de 40 a 70 mil portugueses sofrem de epilepsia, podendo esta iniciar-se em qualquer idade, mas é mais comum até aos 25 e depois dos 65 anos (LPCE, 2005).

Existem uma série de causas para o surgimento da epilepsia. Neste sentido, os autores Acharya, Hattiangady e Shetty, (2008), Herranz (2007), Ottman et al (1998) e Sepúlveda (2000) destacam algumas causas de cariz neonatais, tais como a asfixia no nascimento, o traumatismo perinatal com hemorragia intracraniana, malformação congênita do sistema nervoso central, causas metabólicas, causas tóxicas, infecções do sistema nervoso central , infecção 
extra-encefálica, doença vascular do sistema nervoso central ecausa desconhecida (origem genética ou origem não genética).

A partir disto existem então duas formas de crises epilépticas, as generalizadas que envolvem descargas em todo o cérebro, e as parciais, em que apenas uma zona do cérebro é atingida.

As crises general izadas ocorrem quando a atividade elétrica anormal que causa o ataque epiléptico envolve ambos os lados do cérebro de uma vez só. Dentro deste tipo existem cinco subtipos, sendo eles: (1) os ataques de ausência que fazem parte de uma breve perda de consciência por muitos (entre cinco a vinte) segundos. Geralmente, ocorrem muitas vezes por dia, durante todos os dias e são frequentemente acompanhados de vibração palpebral, estalidos com os lábios ou movimentos de mastigação; (2) os ataques mioclónicos - que têm como parte integrante impulsos ou contrações que parecem choqueis em diversos músculos, geralmente nos braços e pernas. Cada ataque mioclónico pode durar uma fração de segundos, ou um segundo no máximo; (3) os ataques atônicos que consistem na perda repentina de tónus muscular resultando numa queda. Um ataque destes dura poucos segundos e pode ser precedido de um ataque mi ocl ônico muito breve; (4) os ataques clônicos - consistem no súbito enrijecimento dos membros ou de todo o corpo, levando a uma queda. N ormalmente termina após cinco a dez segundos; (5) os tônico-clônicos - que se caracterizam por uma súbita queda (com ou sem grito), rigidez, convulsões, paragem da respiração, face arroxeada, mordedura da língua e/ ou perda de urina, tudo durante menos de 5 minutos. Mais tarde, reaparece a respiração, sendo possível a sensação de confusão el ou fadiga antes do retorno à normalidade (APPLETON; BEIRNE; CHAPPELL, 2000; LPCE, 2005).

Relativamente aos ataques parciais, estes ocorrem quando a atividade elétrica anormal tem início num dos hemisférios, ou num lobo dos hemisférios. Nestes ataques, o nível de consciência da pessoa ou o seu estado de alerta não é afetado durante o ataque. A maioria dos ataques parciais envolve apenas uma mudança de sensação, tal como um cheiro ou gosto (frequentemente desagradáveis ou estranhos), um medo inexplicável, ou até um formigueiro e adormecimento ao nível do rosto ou num dos braços. Já os ataques complexos afetam a consciência ou estado de alerta da pessoa, esta ao ter o ataque parece estar confusa e mesmo agir de modo estranho. Leva a uma paragem seguida de movimentos mastigatórios e automatismos, gestos desajeitados, e o afetado pode ainda deambular. A crise tem a duração de al guns minutos e há al guma confusão após a crise que pode ser mais longa e amnésica que o ataque (APPLETON; BEIRNE; CHAPPELL, 2000; CHOI; KOH, 2008; LPCE, 2005).

No que diz respeito ao diagnóstico é fundamental que este seja estabelecido corretamente, já que sem torna-se impossível ao médico tomar decisões relativamente aos exames que serão necessários e posteriormente decidir qual o tratamento mais indicado. Para se diagnosticar epilepsia é necessário ter 
em conta inúmeros fatores, visto que não existe um teste absoluto que confirme ou negue a epilepsia. Os testes mais usados para diagnosticar são o EEG e a tomografia, em queo primeiro permiteinformação adicional útil, particularmente sobre o tipo deepilepsia ou de síndromeepiléptica (ACHARYA; HATTIANGADY; SHETTY, 2008; APPLETON; BEIRNE; CHAPPELL, 2000; PASTOR et al. 2006) enquanto que o segundo, através da tomografia TC, que usa raios $X$, e da tomografia RMI que usa magnetismo (APPLETON; BEIRNE; CHAPPELL, 2000).

Decorrente dos dados mencionados anteriormente, torna-se explicita a relevância do envolvimento da família neste tipo de problemática, sendo que o papel dos pais acaba por ser um dos aspectos cruciais da integração destas crianças, visto que são os primeiros agentes da intervenção educacional (FON SECA, 1995; PIMENTEL, 2004).

Quando os filhos apresentam este tipo de problemática, os pais acabam por adotar em norma uma atitude marcada pelo excesso de vigilância, ansiedade e super proteção. Geralmente, esta atitude ocorre quando a epilepsia já se encontra bem controlada e pode prolongar-se atéà idade adulta, provocando consequências bastante negativas nos filhos, como baixa autoestima, isolamento social, dificuldades de relacionamento e infantilidade (CAM POS, 2002). É preciso ter em conta que as preocupações, ansiedades e frustrações, vividas ao longo do processo são potenciadas também por pressões vindas de todos os quadrantes, quer de uma sociedade patologizante que põe em risco o desenvolvimento equilibrado dos seus membros e aumenta as dificuldades da educação (MARCHAND; PINTO, 1997).

Para combater este estado de ansiedade excessiva é fundamental que os pais clarifiquem as suas dúvidas, recorrendo à ajuda de técnicos de saúde, visto que um conhecimento mais aprofundado acerca da doença é uma boa estratégia para afastar al guns fantasmas. Quanto mais positiva for a postura dos pais mais facilmente serão capazes de encorajar o filho a ter uma atitude positiva face a si mesmo e à doença e é este suporte emocional que vai precisamente permitir à criança uma melhor qualidade de vida adaptada às suas necessidades (CAMPOS, 2002).

Relativamente ao âmbito escolar, esta problemática não se resume a problemas de aprendizagem, mas sim de discriminação que a criança epiléptica enfrenta levando ao estabelecimento de entraves à educação. A criança com epilepsia, além de sofrer com os problemas neurológicos causados pela doença pode ter enfrentar, no decorrer da sua vida, um obstáculo difícil de transpor, o de ser social mente estigmatizada, já que as eventuais convulsões ou crises de um epiléptico (FERNANDES; SOUZA, 2001; MULAS et al. 2006).

Para eliminar precisamente estas ideias e pôr fim à discriminação de que muitas vezes é alvo a pessoa epiléptica, a difusão de informações acerca deste tema é essencial, de maneira a elucidar de forma mais exata a população em geral, neste sentido a escola assumirá um papel de relevo, sendo uma das 
vias mais adequadas e eficazes de divulgação (ROSA, 1997). De um modo geral, quanto mais informações e conhecimentos os professores e escolas tiverem, mais compreensão haverá, e isso vai ajudar não apenas a criança epiléptica, mas todas as crianças que, eventualmente convivam com crianças com esta problemática, deste modo isto também possibilitará um maior suporte emocional da criança.

Os pais voltam a evidenciar um papel crucial, pois serão eles a elucidar o professor acerca do problema do filho, podendo, por exemplo, explicar a forma como seapresentam os ataques e o respectivo tempo de duração, quais os cuidados imediatos a ter, qual a periodicidade das crises epilépticas e informar também se o filho está si nalizado ea ser acompanhado, se toma fármacos e se existem efeitos colaterais destes mesmos fármacos.

Para que preval eça um bom desenvolvimento escolar e seu respectivo sucesso, será então importante construir esta aliança entre pais e agentes integrantes da escola, de forma a permitir que a criança se sinta aceite no meio escolar, em que predomine a igualdade de direitos.

$\mathrm{Na}$ identificação de crianças com necessidades educativas especiais deverão ser indicados os profissionais da acção social e da saúde, nomeadamente os que prestam cuidados primários, que se encontram em posição ideal para detectar, de forma precoce, situações de risco, atrasos de desenvolvimento ou deficiência (ANIP, 2007).

A Intervenção Precoce (IP) pode definir-se como a prestação de apoio a crianças, atéaos seis anos, em situação dealto risco ou com deficiência etambém às suas famílias, de forma a influenciar a interação familiar e o seu desenvolvimento (ANIP, 2007). Deste modo, a IP consiste em atividades de estimulação dirigidas a crianças e de orientações dirigidas aos pais, que são implementadas como consequência direta e imediata da identificação de um problema de desenvolvimento, ou seja, a IP diz respeito não apenas à criança, mas também aos pais, à família e ao seu ambiente alargado.

A avaliação global da criança implica a identificação dos aspectos de saúde, das suas capacidades e limitações e da avaliação das características comportamentais. A identificação das necessidades, competências, recursos e prioridades das famílias, é facilitada pelos contatos sequenciais com estas, os quais permitem a partilha gradual da informação e a redução do sentimento de intrusão.

A definição de objetivos, estratégias e atividades resulta do consenso entre os profissionais e famílias. A avaliação do plano é um processo dinâmico que permite adaptar o plano de intervenção à evolução da criança e da família e esta avaliação não impede uma avaliação informal que possibilita aferir, com oportunidade, aspectos que se mostrem inadequados em cada novo contato/ visita/ sessão (ANIP, 2007). 
Os principais objetivos da IP visam criar condições facilitadoras do desenvolvimento das crianças, para que este se processe, o mais possível, dentro das fases características dos grupos etários respectivos, minimizando problemas e prevenindo efeitos secundários. Para além disto, tem o objetivo de procurar optimizar as condições de interação criança-família através do reforço de conhecimentos e competências dos pais/ família para lidarem com os problemas dos filhos, mel horando a sua capacidade para identificar eutilizar os seus próprios recursos e os da comunidade, bem como envolver a comunidade no processo de intervenção, de forma contínua e articulada, otimizando os recursos existentes e as redes formais e informais de inter-ajuda.

A IP tem como base o envolvimento da família e a sua participação em todo o processo de intervenção, cabendo-lhes as tomadas de decisão sobre as suas crianças, permitindo um atendimento em que a avaliação e a intervenção sejam contínuas. Caracteriza-se por avaliar as necessidades, prioridades e recursos, conjuntamentepel os técnicos da equipa efamílias, desenvolver um plano conjugado e baseado nessa avaliação, assim como prestar apoio técnicopedagógico e terapêutico de acordo com as necessidades de cada criança/ família.

A intervenção assenta em programas individualizados desenvolvidos nos locais onde as crianças habitualmente se encontram (amas, creches, jardins de infância, etc.) e nas salas da IP (estimulação global e fisioterapia) quando o serviço prestado nas condições anteriores não é considerado útil, suficiente ou exequível. A equipa inclui normalmente profissionais do âmbito da Educação, da Psicologia, do Serviço Social, da Fisioterapia, da Medicina, entre outros (ANIP, 2007).

O Plano de Intervenção é um dos elementos chave da IP nas crianças com necessidades educativas especiais. É precedido pelos primeiros contatos com a família, elabora-se a partir do conhecimento dos problemas e depende do trabalho de colaboração e parceria entre famílias e equipa (ANIP, 2007). O conhecimento sobre a problemática poderá decorrer de materiais como o PIAF (Plano Individualizado de Apoio à Família), o PEI (Programa Educativo Individual) e o PII (Programa de Intervenção Individual) que envolvem aspectos como: informação acerca da criança e da sua história de vida, resultados da avaliação global da criança, identificação das necessidades, competências e recursos familiares e comunitários e das prioridades de intervenção; definição de objetivos, estratégias e atividades; definição dos apoios necessários, inicio e duração prevista; avaliação sistemática do plano de intervenção e eventual reformulação.

Segundo Bailey e Simeonsson (1990), no plano de individual de intervenção traçado por uma equipa pluridisciplinar devem constar os seguintes elementos: (1) o diagnóstico global da situação da criança com este tipo de necessidades educativas especiais no seu contexto de vida, incluindo os aspectos identificativos do seu estado de saúde, das suas competências e capacidades assim como as características comportamentais; (2) a identificação dos recursos e 
necessidades da criança da família, efetuada com esta partilhando informação e através também do inventário das necessidades da família; (3) a designação de apoios a prestar, consensuais entre a equipe pluridisciplinar ea família, mediante a informam já retida; (4) a data de início do plano e o período de duração da intervenção; (5) a periodicidade da avaliação; (6) os procedimentos que permitam um acompanhamento do processo de transição da criança para o contexto educativo formal, neste caso para a pré-escolar; (7) a referenciação das crianças e posterior seleção dos casos com necessidade de apoio é feita às equipas, por solicitação da família, por profissionais de saúde, de educação e da ação social, bem como profissionais de outros serviços.

A intervenção desenvolve-se de forma contínua e integrada, otimizando recursos existentes e criando redes formais e informais de interajuda. Desta forma, as presentes orientações estabelecem os princípios e as condições para o apoio integrado no âmbito das necessidades educativas especiais em regime de intervenção precoce dirigida a crianças com epilepsia. Este plano de intervenção centrado na criança e na família, baseada em ações preventivas e habilitativas, visa, essencialmente, assegurar condições que facilitem o desenvolvimento da criança com epilepsia, potenciar a melhoria das interações familiares, reforçar competências familiares como suporte da sua evolutiva capacitação e autonomia face a esta desordem neurológica.

A infância constitui um período especial mentevalioso para reconhecer os sinais de alarme sobre problemas cognitivos e emocionais ligados à epilepsia, quer ao nível da integração, quer do rendimento escolar, já que quer através da informação dos pais como dos educadores, obtemos as chaves para detectar, intervir e monitorizar a evolução destes problemas (MULAS et al. 2006). A intervenção precoce neste tipo de problemática deverá incidir em crianças que se encontrem entre os 3 e os 6 anos e que apresentem um risco de atraso grave do desenvolvimento, ou seja, naquelas crianças que, manifestem fatores pré, peri, ou pós-natais ou ainda razões que limitem a capacidade de ter um bom aproveitamento no que concerne a experiência importantes de aprendizagem e que pode constituir uma possibilidade de uma ou mais disfunções ocorrerem.

A complexidade queesta desordem coloca ao desenvolvimento global das crianças e à dinâmica familiar exige um processo integrado de atuação que requer o envolvimento da família, o trabalho de equipa e um plano educativo individual de intervenção. Relativamente aos eixos interventivos estes incidem no envolvimento da família em todas as fases do processo de intervenção pelo fato de ser nos primeiros anos que é estabelecido o processo de vinculação determinante no desenvolvimento de padrões adequados de interação entre pais e a criança epiléptica.

É também função da família tomar decisões sobre assuntos que Ihes afetam diretamente por isso é necessário que esta tenha competências especificas ou capacidades a ser desenvolvidas, constituindo isto parte integrante do leque 
de solução para o problema, é importante incutir à família um sentimento de autonomia para mobilizar e utilizar os recursos que necessita contribuindo desta forma para o sucesso da intervenção (PIMENTEL, 2004). Quanto ao trabalho de equipa estedeveser orientado no sentido de responder às necessidades específicas da criança com necessidades educativas especiais e à respectiva família, reforçando o seu envolvimento, autonomia e capacidade para tomar decisões. Esta atuação deve basear-se em relações de confiança entre profissionais e familiares e no respeito pela privacidade, valores e dinâmicas próprias de cada família, deve utilizar o modelo que melhor se adequa às exigências deste tipo de intervenção e que permita uma atuação integrada dos apoios às múltiplas necessidades da criança e da família, por isso torna-se necessário avaliar em conjunto com as famílias necessidades, prioridades e recursos assim como construir um plano individual de intervenção baseado nesta avaliação.

Outro aspecto que deve ser focado neste tipo de intervenção é a nomeação de um responsável de caso, que tem como principal função garantir a articulação dos apoios prestados. Atuar no domicílio e em ambientes onde a criança habitualmente se encontra (pré-escola) também se torna fundamental, da mesma forma que a partilha de informação entre os membros da equipe de uma forma sistêmica se torna crucial. Em situações específicas verificadas no quadro clínico destas crianças pode ser necessário recorrer a apoios complementares, como terapias.

\section{Conclusão}

A epilepsia é uma doença que pode surtir diversos efeitos nos portadores, a nível neurológico, escolar, familiar e/ ou social, podendo causar mazelas graves se não ocorrer uma intervenção adequada neste âmbito. A intervenção nesta perturbação implica uma cultura e atitude ativa da equipa envolvida, baseada no reconhecimento de que as necessidades destas crianças só podem ser devidamente avaliadas e interpretadas no contexto familiar e social. Baseia-se numa lógica de ação social, implicando uma aproximação com a comunidade permitindo um melhor conhecimento das necessidades, dos problemas e dos recursos e exige uma atuação assente em programas individualizados, desenvolvidos em ambientes onde a criança se encontra habitualmente.

Um plano de intervenção bem organizado é essencial nestes casos, de modo a permitir um maior conforto e à vontade com a doença, da pessoa epiléptica e correspondente família (SANTOS; SANCHES, 2004). Deste modo, os objetivos de intervenção deverão basear-sena criação de condições facilitadoras do desenvolvimento global da criança, minimizando problemas ou risco de atraso do desenvolvimento, de forma a prevenir possíveis sequelas na otimização das condições de interacção entre a criança e a família. 
A finalizar é importante voltar a referir a importância que assumem os terapeutas no tratamento de doentes epilépticos. Estes devem ter em conta as preocupações e sentimentos da criança epiléptica, assim da restante família, que muitas vezes depara-se com bastantes dificul dades em aceitar a condição de uma criança que exige cuidados no seu dia-a-dia, desencadeando desta forma, tal como na criança, sentimentos de sofrimento e constante angústia. Para que a intervenção se desenrole com sucesso, será essencial criar laços de empatia com a criança e com a família para que ocorra um sentimento de compreensão e por conseguintenão existam resi stências que possam por em causa todo o tratamento.

É ainda de enorme importância que se continue a investir e investigar nesta área, procurando também formas alternativas de sensibilizar e intervir, com o intuito de elucidar as pessoas acerca desta problemática, de modo a que possa ocorrer uma maior conscientização e compreensão da comunidade.

\section{REFERÊnCIAS}

ACHARYA, M.; HATTIANGADY, B.; SHETTY, A. Progress in neuroprotectivestrategies for preventing epilepsy. Prog N eurobiol, v. 84, n. 4, p. 363-404, 2008.

ASSOCIAÇÃO NACIONAL DE INTERVENÇÃO PRECOCE (ANIP). Consulta de desen volvimento na comunidade. Braga: Fundação CalousteGulbenkian, 2006.

APPLETON, R.; CHAPPELL, B.; BEIRNE, M. Tudo sobre epilepsia. São Paulo: Andrei Editora, 2000.

ASSOCIAÇÃO FAMALICENCE DE PREVENÇÃO E APOIO À DEFICIÊNCIA. Serviço de interven ção precoce: relatório de transição para o jardim de infância. Vila N ova de Famalicão: Associação Famalicence de Prevenção eA poio à Deficiência, 2006a.

ASSOCIAÇÃO FAMALICENCE DE PREVENÇÃO E APOIO À DEFICIÊNCIA. Plano individualizado de apoio à família. Vila Nova de Famalicão: Associação Famalicence de Prevenção e A poio à Deficiência, 2006b.

BAILEY, L.; SIMEONSSON, P. Recursos institucionais de apoio à família. EUA: University of North Carolina, 1990.

CAMPOS, A. A família e a epilepsia. Disponível em http:/ / www.educare.pt/ educare. [Consultado em 14 A bril de 2008], 2002.

$\mathrm{CHOI}$; J.; $\mathrm{KOH}$, S. Role of brain inflammation in epileptogenesis. Y onsei M ed J , v. 49, n. 1, p.118, 2008.

FERNANDES, P.; LI, L. Percepção de estigma na epilepsia. Journal of epilepsy and clinical neurophysiology, vol. 12, n. 4, p. 207-218, 2006.

FERNANDES, P.; SOUZA, E. Procedimento educativo na epilepsiainfantil. Estudos deP sicologia, v. 6, n. 1, p. 115-120, 2001.

FON SECA, V. Educação especial - programa deestimulação precoce. Porto A legre: Artmed Editora, 1995. 
GOLOMB, M. et al. Perinatal strokeand therisk of developing childhood epilepsy. J Pediatr, v.151, n.4, p.409-413, 2007.

GOMES, A . A final ésó E pilepsia. Porto: Âmbar, 2007.

HERRANZ, J.L. Repercusión cognitiva delas epilepsias precoces. Revista deN eurología, v. 44, n. S3, p. S43-S45, 2007.

KIRK, S.; GA LLA GHER, J. Educação da criança excepcional. São Paulo: MartinsFontes, 2002.

LIGA PORTUGUESA CONTRA A EPILEPSIA (LPCE) [em linha]. Disponível em http:/ / www.lpce.pt/ . [Consultado dia 05 de A bril de2009].

MARCHAND, H.;PINTO, H. Família- contributos da psicologia e das ciências da educação. Lisboa: Editora Educa, 1997.

MULAS, F. et al. Dificultades del aprendizaje en los niños epilépticos. R evista deN eurología, v. 42, n. S2, p. S157-S162, 2006.

NIELSEN, L. B. N ecessidades educativas especiais na sala de aula. Porto: Porto Editora, 1999.

OTTMAN, R.; LEE, J.; HAUSER, A.; RISCH, L. Are generalized and localization-related epilepsies genetically distinct?. A rch N eurol, v. 55, n. 3, p. 339-344, 1998.

PASTOR, J. et al. Bases fisiopatológicas de la epilepsia del lóbulo temporal: estudios en humanos y animales. Revista de N eurología, v. 42, n. 11, p. 663-673, 2006.

PIMENTEL, J. S. Intervenção focada na família: desejo ou real idade. Lisboa: Secretariado N acional para a Reabilitação eI ntegração das Pessoas com Deficiência, 2004.

ROSA, M. Obstáculos percebidos por pais e professores no atendimento das necessidades de crianças com epilepsia. R evista latino-am.enfermagem, v. 5. p. 37-44, 1997.

SEPÚLVEDA, F. M anual deepilepsia. Rio deJaneiro: Colina Editora, 2000.

Recebido em: 18/ 05/ 2009

Reformulado em: 30/ 10/ 2009

A provado em: 04/ 11/ 2009 